\title{
Mutations in Salmonella typhimurium Conferring Resistance to Felix O Phage without Loss of Smooth Character
}

\author{
By D. G. MACPHEE*, V. KRISHNAPILLAI†, R. J. ROANTREE \\ AND B. A. D. STOCKER \\ Department of Medical Microbiology, Stanford University School of Medicine, \\ Stanford, California 94305, U.S.A.
}

(Received 24 April 1974; revised 14 October 1974)

\begin{abstract}
SUMMAR Y
Several mutants obtained from smooth Salmonella typhimurium strains by selection for resistance to Felix $O$ (FO) phage [whose receptor site includes the $\mathrm{N}$-acetylglucosamine branch of the lipopolysaccharide (LPS) core] were smooth in cultural properties, antigenic character and phage sensitivity pattern (except for their FO resistance). However, the affected genes of several such 'FOR' (FOresistant) mutants were shown by transduction to map in the short cysE-pyrE segment, which includes nearly all known $r f a$ genes responsible for synthesis of LPS core. All of seven FOR mutants differed from their parents, and resembled $r f a$ mutants with defects in the deeper part of the LPS core, by increased sensitivity to various antibiotics. One FOR mutant was non-virulent $\left(\mathrm{LD}_{50}>10^{7}\right.$, compared with $<$ IOO for its parent); LT7 derivatives given this FOR gene by co-transduction with $c y_{s} E^{+}$were likewise non-virulent. It is inferred that FOR mutations affect the assembly of the inner part of the LPS core, perhaps causing incomplete blocks in glycosyl transferase reactions.
\end{abstract}

\section{INTRODUCTION}

Felix $\mathrm{O}$ phage (hereinafter called FO) attacks nearly all smooth strains of Salmonella, whatever their $\mathrm{O}$ antigen, but is active on very few strains of Escherichia coli, etc. (Kallings, I967). In Salmonella typhimurium it attacks not only smooth strains but also some nonsmooth mutants: those unable to transfer $O$ chains from their site of synthesis to the 'complete' lipopolysaccharide (LPS) core, through mutation at $r f a L$ or $r f b T$; the semirough (SR) class $r f c$, deficient in polymerization of $O$ repeating units; and classes $r f b$ and pmi, unable to synthesize O repeating units (Wilkinson, Gemski \& Stocker, 1972). Phage FO, however, does not attack $r f a$ mutants of various classes in which synthesis of the LPS core is defective. Lindberg and his colleagues (for review see Lindberg, I973), from host range and from measurements of rates of adsorption by whole bacteria and of neutralization by isolated LPS, infer that the $N$-acetylglucosamine side-branch which is attached to the glucose II unit of the complete LPS core (Fig. I) is an essential part of the adsorption site of FO phage. Most mutants selected from smooth strains by application of FO phage are of type $r f a$, with various sorts of defects in LPS core structure, and are recognizable as such by sensitivity to different combinations of rough-specific phages and/or to bile salts and by total or partial loss of 'smooth' cultural characters, $O$ antigen(s) and sensitivity to smoothspecific phages (Wilkinson et al. 1972). We here describe FO-resistant mutants of smooth

* Present address: Department of Genetics, LaTrobe University, Bundoora, Victoria, Australia.

+ Present address: Department of Genetics, Monash University, Clavton, Victoria, Australia.

Vol. 86, No. 2 was issued 20 February 1975 


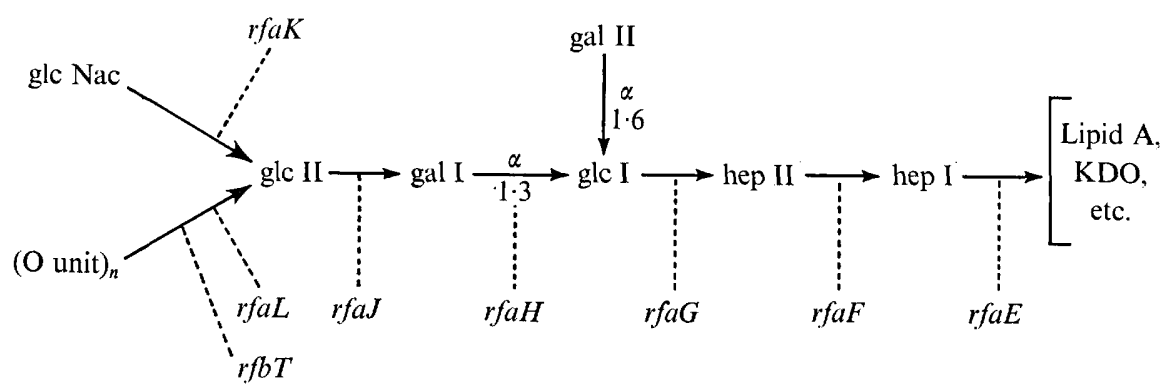

Fig. I. Main features of structure of Salmonella LPS and symbols assigned to genes concerned with formation of various units or bonds. glcNac, $N$-acetylglucosamine; glc, glucose; gal, galactose; hep, heptose; KDO, ketodeoxyoctonate; $n$, no. of repeating units in $\mathrm{O}$ chain. Heptose and phosphate branch(es) in heptose region of core omitted.

S. typhimurium strains which retain all (or nearly all) the ordinary properties of smooth strains. However, genetic analysis of four mutants indicates that the mutations concerned lie in the $c y s E-p y r E$ region of the linkage map, a short segment which includes nearly all the $r f a$ loci so far identified (Kuo \& Stocker, I972; Lehmann et al. 1973), and for this and other reasons it seems likely that the characteristic phenotype (termed FOR, for FO-resistant) of these mutants results from some anomaly, of unknown nature, in biosynthesis of the LPS core.

\section{METHODS}

Media. The nutrient agar used was Oxoid blood agar base No. 2 (code CM55), which contains $0.5 \%(\mathrm{w} / \mathrm{v}) \mathrm{NaCl}$. Phage FO, whose adsorption is greatly affected by the cationic environment (Lindberg \& Holme, I969), does not plate well on media of low cation content (e.g. Difco nutrient agar) unless such media are supplemented with a salt.

Bacteria. Strains are listed in Table I. Most of the FOR mutants were obtained by phage selection from two mouse-virulent genetically marked sublines of an S. typhimurium strain, M747I, which is of type (or subspecies) FIRN (Morgenroth \& Duguid, 1968). The two M747 I sublines used as parent strains, TV253 and SL4522, are isogenic except that they have different cys and his mutations (MacPhee \& Stocker, 1969; Krishnapillai, MacPhee \& Stocker, I97I). For the origin of FO phage and of other phages used to determine LPS character see Wilkinson et al. (I972). Phage P22.L4 (i.e. int-, unable to lysogenize) was used for transduction.

Phage and genetic methods. Phages were propagated by the soft agar layer method, generally on LT2 sublines known to be 'cured' of prophage Fels 2, carried by LT2 wild-type (Wilkinson et al. 1972). Counts of plaque-forming units (p.f.u.) were made by the drop-onlawn method (Gemski \& Stocker, 1967). Patterns of sensitivity to smooth-specific, roughspecific and other phages were determined as described by Wilkinson et al. (I972). Mutants resistant to FO or P22.c2 phage were selected by application of drops of phage to nutrient agar plates surface-inoculated from broth cultures, and colonies growing in areas of lysis were purified by re-isolation from single colonies before tests of phage sensitivity, etc. To obtain $c y s E^{+}$or $p y r E^{+}$transductants, plates of defined medium inoculated by flooding with an overnight broth culture of the (smooth) auxotrophic recipient were dried, then spotted with drops (about $0.0 \mathrm{I} \mathrm{ml}$ ) of $\mathrm{P}_{22}$. $\mathrm{L}_{4}$ lysates with titres of about $\mathrm{IO}^{10}$ p.f.u./ml. The prototrophic transductants arising were purified by single-colony re-isolation on selective medium before testing for phage sensitivity, etc. 


\section{Table I. Main strains}

All strains are $S$. typhimurium, and derived from strain LT2, LT7 or M747I ; the latter is of type FIRN (Morgenroth \& Duguid, 1968 ), of phage-type $\mathrm{I} / \mathrm{I}$, and mouse-virulent $\left(\mathrm{LD}_{50}\right.$, by intraperitoneal route, < 100 bacteria).

\begin{tabular}{|c|c|c|c|}
\hline Strain no. & Genotype & Description* & References \\
\hline \multicolumn{4}{|c|}{ Starting strains } \\
\hline pyrEI25 & LT2 pyrEI25 & Recipient for transduction & Kuo \& Stocker (1972) \\
\hline $\operatorname{ser} A I_{3}$ & $\mathrm{LT} 2 \operatorname{ser} A I_{3}$ & FO-sensitive parent of SA28 & Sanderson \& Saeed (1972) \\
\hline SL1027 & \multicolumn{2}{|c|}{$\begin{array}{l}\mathrm{LT} 2 \text { metA22 } \operatorname{trpB2} \mathrm{HI-b} H 2-e, n, x \text { FO-sensitive indicator strain } \\
\text { 'cured of Fels } 2 \text { ' flaA66 strAI2O } \\
x y /-404 \text { metE55I }\end{array}$} & $\begin{array}{l}\text { Wilkinson et al. (1972), } \\
\text { Kuo \& Stocker (1972) }\end{array}$ \\
\hline SLI I 83 & As sLio27, but $r f a F_{546}$ & $r f a F$ parent of sL 4548 & Wilkinson et al. (1972) \\
\hline SLI 547 & LT7 cysE3o gal-85I & Recipient for transduction & Kuo \& Stocker (1972) \\
\hline SL4522 & $\begin{array}{l}\text { M747 I (ColEI -30) leu-IO5I } \\
\text { malB479 cysiII } 73 \text { hisC } 527 \\
\text { (amber) }\end{array}$ & $\begin{array}{l}\text { FO-sensitive parent of SL4504 } \\
\text { and SL4505 }\end{array}$ & MacPhee \& Stocker ( $19 \epsilon_{9}$ ) \\
\hline TV253 & $\begin{array}{l}\text { M747I (ColEI-30) leu-105I } \\
\text { malB479 hisCIr5o cysGir75 }\end{array}$ & $\begin{array}{l}\text { FO-sensitive parent of strains } \\
\text { SL4804 to SL } 4808\end{array}$ & $\begin{array}{l}\text { Krishnapillai et al. (197I), } \\
\text { Subbiah (I963) }\end{array}$ \\
\hline \multicolumn{4}{|c|}{ FOR mutant strains } \\
\hline $\mathrm{SA} 28+$ & LT2 $\operatorname{ser} A I_{3} \mathrm{~F}^{+} r f a-3058$ & $\begin{array}{l}\mathrm{F}^{+} \text {derivative of } \operatorname{ser} A I_{3} \\
\text { found FOR }\end{array}$ & Sanderson \& Saeed (1972) \\
\hline SL4504 & As TV253, but $r f a-663$ & FO-selected, found FOR & - \\
\hline SL4505 & As TV253, but $r f a-664$ & FO-selected, found FOR & 一 \\
\hline SL4548 & As SLI I 83 , but $r f a-68 I$ & $\begin{array}{l}\text { Bile salt-resistant 'revertant', } \\
\text { found FOR }\end{array}$ & - \\
\hline SL4804 & As SL4522, but $r f a-704$ & FO-selected, found FOR & - \\
\hline SL4805 & As SL4522, but $r f a-705$ & FO-selected, found FOR & - \\
\hline SL4806 & As SL4522, but $r f a-706$ & FO-selected, found FOR & - \\
\hline SL4807 & As SL4522, but $r f a-707$ & FO-selected, found FOR & - \\
\hline SL.4808 & As SL4522, but $r f a-708$ & FO-selected, found FOR & - \\
\hline \multicolumn{4}{|c|}{ Derivatives of FOR strains } \\
\hline SL451 2 & As SL 4505 , but $r f c-67 I$ & $\begin{array}{l}\text { P22-resistant mutant of FOR } \\
\text { strain SL4505: found FO- } \\
\text { sensitive, phage pattern Zsr, } \\
\text { inferred to be } r f c \text { mutant }\end{array}$ & Lindberg \& Svensson (1975) \\
\hline
\end{tabular}

* FOR: smooth in phage sensitivity pattern but resistant to FO phage (see text). $r f a F$ : class of mutant deficient in addition of heptose 11 unit of LPS core. Zsr: phage sensitivity pattern typical of SR strains, with LPS core bearing single $O$ units instead of $O$ chains.

$\uparrow$ The FOR property of this $\mathrm{F}^{+}$strain was originally detected in its $\mathrm{HfrK}_{3}$ derivative, sa486 (Sanderson et al. 1972) and the latter strain was used for conjugational mapping of the mutation causing FOR character.

Antibiotic sensitivity tests. Plates of Oxoid sensitivity test medium (agar) (CM2 I5) containing graded concentrations of various antibiotics (Table 2) were inoculated by multiprong replication from bacterial suspensions prepared by $\mathrm{I} / \mathrm{I} 000$ dilution into saline of overnight cultures in brain heart infusion broth (Difco); the inocula thus delivered contained between 100 and 300 cells. The lowest concentration of antibiotic preventing formation of any colonies during overnight incubation (or reducing the number of visible colonies to $<\mathrm{IO}_{\%}^{\circ}$ of that on control plates without antibiotic) was taken as the minimal inhibitory concentration (m.i.c.). 
Mouse virulence, clearance after intravenous inoculation and serum sensitivity tests. For virulence tests random-bred CFI mice were inoculated intraperitoneally (Krishnapillai, 197I). For clearance tests inocula of about $10^{8}$ bacteria, made up of a mixture of two genetically marked strains, were injected into a caudal vein, and their removal from the circulation was followed by viable counts on blood samples taken at short intervals from the retro-orbital plexus (Krishnapillai, I971). Sensitivity to the bactericidal effect of normal human serum was tested as described by Steward, Collis \& Roantree (I 966).

\section{RESULTS}

\section{Isolation and cultural properties of FOR mutants}

Two FOR (i.e. FO-resistant but apparently smooth) mutants, SL4504 and SL4505, were encountered amongst a set of FO-resistant mutants isolated from strain TV253 (Krishnapillai et al. I97I). One of the mutants, SL4505, had the unexpected property of nonvirulence. Selection for FO resistance yielded an additional five FOR mutants, SL4804 to SL4808, in another mouse-virulent subline, SL4522, derived from the same $S$. typhimurium ancestor as TV253. These five FOR mutants, unlike SL4505, retained mouse virulence. Two sublines of FOR phenotype were encountered by chance amongst stocks derived from $S$. typhimurium strain LT2. Several Hfr derivatives (Sanderson, Ross, Ziegler \& Mäkelä, I972) of LT2 serAI 3 and also their immediate parent, SA28 ( = LT2 $\left.\operatorname{serAI} 3 \mathrm{~F}^{+}\right)$, were of phenotype FOR, but LT2 serAI3 itself was smooth and FO-sensitive. Another FOR derivative of LT2, strain SL4548, was isolated as a bile-salt-resistant 'revertant' from SLI I 83, a strain with a $r f a F$ mutation preventing addition of the second heptose unit of the core and causing sensitivity to bile salts (Wilkinson et al. 1972).

All the FOR strains gave smooth-looking colonies on nutrient agar, uniform growth in broth and normal slide agglutination in anti- $\mathrm{O}_{4}$ and anti-O5 sera. In phage pattern tests (Wilkinson et al. 1972) all the FOR mutants were lysed by the smooth-specific phages, P22.c2, P22h.c2 and 9NA, and P22.c2 plated with an efficiency of about one on all of them. All the FOR mutants were resistant to FO phage, in that application of phage at about $\mathrm{IO}^{8}$ p.f.u./ml produced no plaques and no visible thinning of growth; however, phage at $10^{10}$ p.f.u./ml sometimes produced a few plaques or 'thinning' of SL4504 and SL4505. It should be noted that FO phage, even after propagation on TV253, plates with an efficiency of only about I \% on strain TV253 (and other smooth sublines of S. typhimurium $\mathrm{M} 747 \mathrm{I}$ ) as compared with its titre on SLI027, a smooth LT2 subline. All the FOR mutants were resistant to all six of the rough-specific phages used, except that SL4548 showed partial sensitivity (plaque formation from application of concentrated phage) to phages Ffm and Br6o. However, as this strain was obtained by 'reversion' from a rough parent (of type $r f a F$ ) it is not clear whether these sensitivities result from the mutation which caused the FOR phenotype or from the original $r f a F$ mutation. Phages $\mathrm{P}_{22} . c 2$ and $\mathrm{P} 22 \mathrm{~h} . c 2$ produced more nearly complete clearing in the lawn of growth of several FOR mutants than they did on their parent strains. Discrete colonies appearing in some of these cleared areas and found to be P22-resistant mutants are described below.

On nutrient agar plates incubated overnight at $37^{\circ} \mathrm{C}$ the colonies of two FOR mutants, SL4504 and SL4505, were somewhat smaller than those of their FO-sensitive parent, TV253. No difference in colony size was noted in the other FOR mutants. 
Table 2. Antibiotic sensitivity, co-transduction and virulence of FOR mutants

\begin{tabular}{|c|c|c|c|c|c|c|c|c|c|c|}
\hline \multirow{2}{*}{$\begin{array}{c}\text { Strain } \\
\text { no. }\end{array}$} & \multirow[b]{2}{*}{ Parent } & \multicolumn{6}{|c|}{$\begin{array}{l}\text { Antibiotic sensitivity* } \\
\text { (m.i.c. relative to parent) }\end{array}$} & \multicolumn{2}{|c|}{ Co-transduction $\dagger$} & \multirow{2}{*}{$\begin{array}{c}\text { Mouse } \\
\text { virulence } \\
\text { (survivors; } \\
\text { dose tested) }\end{array}$} \\
\hline & & Bacitr. & Vanco. & Eryth. & Colist. & Nafcil. & Oxacil. & cysE & pyrE & \\
\hline SL45O4 & TV253 & 0.3 & 0.5 & I & 0.7 & NT & NT & $1 / 40$ & $1 / 40$ & $0 / 10(1600)$ \\
\hline SL4505 & TV253 & 0.4 & 0.2 & 0.1 & 0.4 & 0.16 & 0.25 & $\begin{array}{l}28 / 40 \\
4 / 40\end{array}$ & $4 / 80$ & $5 / 5\left(7 \times 10^{6}\right)$ \\
\hline $\mathrm{SL} 4804$ & SL4522 & 0.8 & 0.4 & I & 0.3 & 0.3 & 0.3 & NT & NT & $0 / 3(130)$ \\
\hline SL4805 & SL4522 & 0.8 & 0.6 & 0.8 & 0.4 & $0 \cdot 8$ & 0.8 & NT & NT & $0 / 3(150)$ \\
\hline SL4806 & SL 4522 & 0.8 & 0.6 & 0.8 & 0.4 & 0.7 & 0.8 & NT & NT & $1 / 3(140)$ \\
\hline SL4807 & SL 4522 & 0.8 & $0 \cdot 6$ & $0 \cdot 8$ & 0.4 & $0 \cdot 7$ & 0.8 & NT & NT & $1 / 3(120)$ \\
\hline SL4808 & SL4522 & 0.8 & 0.6 & 0.8 & 0.6 & 0.7 & 0.8 & NT & NT & $0 / 3(140)$ \\
\hline SA 28 & $\operatorname{ser} A 13$ & NT & NT & NT & NT & NT & NT & $4 / 24$ & $3 / 40$ & NT \\
\hline SL 4548 & SLI 183 & NT & NT & NT & NT & NT & NT & $3 / 24$ & $2 / 40$ & NT \\
\hline
\end{tabular}

NT, Not tested.

* Figures are m.i.c. (see Methods) of bacitracin, vancomycin, erythromycin, colistin, nafcillin and oxacillin, relative to m.i.c. for parent strain tested at same time.

$\dagger$ No. of transductants, cys $E^{+}$or $p y r E^{+}$, found FO-resistant/no. of transductants tested, (in experiments on transduction of $c y s E^{+}$or pyrE $E^{+}$by phage $\mathrm{P}_{22}$. L4 to SLI547 (= LT2 cysE30 gal) or LT2 pyrEI25). Cotransduction of the FOR allele $r f a-3058$ was tested using $\mathrm{P}_{22}$. L4 grown on SA486, the HfrK 3 derivative of SA28 (LT2 ser 4 I $3 F-r f a-3058)$.

$\$$ No. of survivors/no. of mice injected, intraperitoneal route; no. of bacteria tested (which was largest dose tested, in case of non-virulent FOR mutant SL4505, and smallest dose tested, in case of all other mutants) are given in parentheses. Each of the FO-sensitive parent strains, TV253 and SL4522, has an LD $_{50}$ of $<100$ bacteria.

\section{Antibiotic sensitivities}

Mutants of various $r f a$ classes, with different defects in LPS core structure, differ from smooth strains, or rough strains making complete core LPS, by changes in sensitivity to various antibiotics (Roantree, Kuo, MacPhee \& Stocker, I 969; Schlecht \& Schmidt, I969). The seven FOR mutants obtained in derivatives of $S$. typhimurium M747I were therefore tested for sensitivity to various antibiotics (see Methods). (The FOR derivatives of LT2 lines were not investigated with respect to antibiotic sensitivity: SL4584 because its immediate parent has a rough mutation itself causing alterations of antibiotic sensitivities; and SA2 8 because it differed from its parent strain, LT2 $\operatorname{serA13}$, by $\mathrm{F}^{+}$character as well as by FOR phenotype.) The m.i.c. of a given drug for a given strain varied slightly between experiments, but some consistent differences in sensitivity between FOR mutant and FO-sensitive parent (always tested simultaneously) were observed. In Table 2, which summarizes observations from several experiments, the m.i.c. of each FOR mutant for each drug is recorded as a proportion of the m.i.c. of its FO-sensitive parent. All seven mutants showed increased sensitivity to bacitracin, vancomycin and colistin, with m.i.c.s in the range 0.2 to 0.8 of those of their parents. One FOR mutant, SL4505, showed about a tenfold increased sensitivity to erythromycin; the other six mutants showed at most a slight increase (m.i.c. $0 \cdot 8$ to $\mathrm{I} \cdot 0$ of control). Two mutants SL4505 and SL4804, were conspicuously sensitive to nafcillin and oxacillin (m.i.c. 0.16 to 0.3 of control); four others tested showed lesser increases in sensitivity (m.i.c. 0.5 to 0.8 of control).

\section{Mapping of rfa mutations}

When an FOR donor strain, SA486 (the $\mathrm{HfrK}_{3}$ derivative of SA28, LT2 serA13 $\mathrm{F}^{+}$) was crossed to various FO-sensitive recipients, 17 of 32 clones selected for the donor rhat. (rhamnose utilization) had its FOR character, as also had all of 20 selected for the donor $c y s E^{+}$. The close linkage to $\operatorname{cys} E$ of the FOR gene of the $\mathrm{Hfr}$ donor suggested that its 
mutation might be in one of the $r f a$ genes (concerned with LPS core structure) located between cysE and pyrE and co-transducible with each of these loci by P22 (Kuo \& Stocker, I972). Stocks of phage P22. L4 grown on FOR mutants SL4504, SL4505, SA486 and SL4548 were used to evoke $c y s E^{+}$transductants from SLI547 (LT7 cysE30 gal) and pyrE $E^{+}$from LT2 pyrEI25; both these recipients were smooth and FO-sensitive. In each cross a minority of the transductants were found to be FOR, like the donor (Table 2). In control crosses none of $40 \operatorname{trp}^{+}$transductants evoked from SLIO27 (LT2 metA $\operatorname{trpB}$, etc., see Table 1) by phage grown on SL4505 had the donor FOR character; and none of 30 cys $E^{+}$transductants obtained from SLI 547 by application of phage grown on TV253 (the FO-sensitive parent of SL4505) was resistant to FO phage. None of these $30 \mathrm{cys} E^{+}$transductants showed the partial resistance (efficiency of plating approx. $\mathrm{IO}^{-2}$ ) characteristic of the donor strain, derived from $S$. typhimurium M747I, and it is therefore unlikely that its incomplete FO sensitivity is determined at an $r f a$ locus between $c y s E$ and $p y r E$. The observed frequency of co-transduction of the FOR determinant of SL4505 with $c y s E$ varied between experiments, for unknown reasons. As mentioned above, the colonies on nutrient agar of FOR mutants SL4504 and SL4505 were smaller than those of their FO-sensitive parent, TV253. This difference was also observed amongst cys $^{+}$transductants obtained from SLI 547 by application of P22.L4 grown on SL4505; in one experiment all of $15 \mathrm{cys} s^{+}$transductants scored as "large colonies' proved to be FO-sensitive, like their recipient parent, whereas I 2 of I 5 transductants scored as 'small colonies' were FOR. (In this experiment all I 2 of the FOR transductants were P22-sensitive, whereas 9 of the 18 FO-sensitive clones appeared P22resistant, presumably by virtue of 'pseudo-lysogeny' for P22. L4.)

To test whether the altered antibiotic sensitivities of FOR mutants were found also in strains made FOR by co-transduction, we tested the antibiotic sensitivities of five cys $E^{+}$ transductants obtained from SL1547 by treatment with P22.L4 grown on FOR mutants SL4504 and SL4505. Two transductants with the FOR character from SL4505 and one with the FOR character of $\mathrm{SL} 4504$ showed all the increased antibiotic sensitivities of their FOR donor parents, whereas two transductants which retained the FO sensitivity of their recipient parent, SLI547, were as sensitive to all the agents tested as the previously investigated FO-sensitive parent strains, TV253 and SL4522.

\section{Mouse virulence and related properties of FOR mutants}

Mutants of type $r f a$, like other rough mutants of $S$. typhimurium, are non-virulent, or at least much less virulent than their smooth parent strains. Since it seemed that the FOR phenotype reflected altered LPS core structure, due to $r f a$ mutation, we thought that FOR mutants also might be of diminished virulence. We tested the mouse virulence of all the FOR mutants isolated from the highly virulent $\left(\mathrm{LD}_{50}\right.$, intraperitoneal route, $<$ 100 bacteria) parents, TV253 and SL4522 (Table 2). One FOR mutant, SL4505, was non-virulent, since all of five mice given $7 \times 10^{6}$ bacteria survived (as also did all mice given smaller inocula). The other six mutants tested were all still virulent, since all or most mice given the smallest challenge dose $(<2000$ bacteria) died. To test whether the non-virulence of SL4505 resulted from its FOR character or from coincidental mutation, we tested six cys $E^{+}$transductants obtained from SLI547 by application of P22. L4 grown on SL4505. All of three FOR transductants were non-virulent, all mice surviving challenge by $10^{6}$ to $2 \times 10^{6}$ bacteria; all of three FO-sensitive transductants were virulent, in that at least one of each group of three mice inoculated with 200 to 300 bacteria died. Thus the avirulence of SL4505 is transduced with its FOR character.

Non-smooth mutants of Salmonella species, unlike most smooth strains, are very rapidly 
removed from the circulation after intravenous inoculation into normal mice (Krishnapillai, 197I). We tested the rate of clearance of the non-virulent FOR mutant, SL4505, in parallel with that of its virulent, FO-sensitive parent, TV253 (which is leu cys his). Each of three mice was inoculated intravenously with $0.2 \mathrm{ml}$ of saline $(0.85 \% \mathrm{NaCl})$ containing equal numbers (approx. $2.5 \times 10^{8}$ ) of a $c y s^{+}$transductant derived from the FOR mutant, and of a $h i s^{+}$ transductional derivative of its FO-sensitive parent. Differential viable counts on blood samples collected from the retro-orbital plexus at $0.5,2,3,4$ and $5 \mathrm{~min}$ after inoculation showed that both the FOR mutant and its FO-sensitive parent were only slowly removed from the circulation, the value of $K$ (phagocytic index) for each strain being $0 \cdot 006 / \mathrm{min}$. In similar experiments, rough derivatives of $S$. typhimurium are rapidly cleared, e.g. 80 to $90 \%$ decrease in viable count in $5 \mathrm{~min}$, giving $K=0.2$ to 0.3 (Krishnapillai, 197I).

Two FOR mutants, SL4504 and SL4505, were tested for susceptibility to killing by incubation in normal human serum (Steward et al. I966). Both proved to be almost completely resistant, the survival of bacteria at about $10^{3} / \mathrm{ml}$ incubated two $\mathrm{h}$ in normal human serum being about $30 \%$, which is the same as the survival of their FO-sensitive parent, TV253. By contrast, various $r f a$ mutants of TV253 were sensitive and gave survivals at $2 \mathrm{~h}$ of $<10^{-3}$, when tested at about $10^{5} / \mathrm{ml}$ in the same experiment.

\section{FO-sensitive mutants obtained from FOR strains by selection for P22-resistance}

As noted above, application of phage P22.c2 of titre $10^{8}$ p.f.u. $/ \mathrm{ml}$, to surface-inoculated plates produced clear areas in the lawns of growth of several FOR mutants (and transductants), in contrast to the areas of incomplete clearing of their wild-type FO-sensitive parent strains. Colonies growing up in such areas of clearing of the FOR mutant, SL4505, were picked, purified and tested for phage sensitivities. Most such clones differed from their FOR parents by resistance to the three O-specific phages used, $\mathrm{P} 22 . c 2, \mathrm{P} 22 \mathrm{~h} . c 2$ and $9 \mathrm{NA}$, and by sensitivity to one or more rough-specific phages (Wilkinson et al. 1972), and were inferred to result from mutation at some $r f$. locus (or at galE or galU or pmi). About half of the P22-resistant mutants of SL4505 proved to be FO-sensitive, unlike their parent which is of phenotype FOR. Most of these FO-sensitive $r f$. mutants isolated from SL4505 were sensitive also to the rough-specific phage P22I, but to no other phage tested. They thus showed the phage-sensitivity pattern termed ' $\mathrm{Zsr}$ ', i.e. resistance to O-specific phages and to roughspecific phages except $\mathrm{P}_{22 \mathrm{I}}$, and sensitivity to FO phage. This pattern is typical of "semirough' (SR) strains (Wilkinson et al. 1972) which make LPS with single O units because of deficiency of $O$ polymerase, determined at $r f c$ (Naide et al. 1965). One such presumed $r f c$ mutant of SL4505, labelled SL45I 2, was used for phage adsorption measurements (Lindberg \& Svensson, 1975). Other FO-sensitive mutants of, SL4505 obtained by selection for P22 resistance were sensitive to several rough-specific phages, giving patterns approximating to the 'R-sensitive' pattern characteristic of mutants making complete core LPS without O-chains (classes $r f b, r f a L$ and $p m i$ ). Some FO-sensitive mutants were also encountered amongst those selected for P22 resistance from the two other FOR strains tested, SL4805 and SL4808.

\section{DISCUSSION}

We suspect that FOR mutants are resistant to Felix $O$ phage because of loss (or diminution) of ability to adsorb this phage. This could not easily be tested, because the rate of adsorftion of FO phage even to smooth FO-sensitive $S$. typhimurium strains is too low to be easily measurable. However, the results of Lindberg \& Svensson (I975) are compatible with the hypothesis that FOR mutants owe their resistance to failure to adsorb. The LPS extracted 
from FO-sensitive Salmonella can bind to and 'trigger' FO phage (Lindberg \& Hellerqvist, 197I) and the $N$-acetylglucosamine branch unit of the glucose II unit of the complete core is an essential part of the phage adsorption site (Lindberg \& Holme, 1969). It therefore seemed likely that FOR mutants had $r f a$ mutations affecting the structure of their LPS core in such a way as to reduce the number, and/or the accessibility to phage, of completed core chains. The inference that FOR mutants make an abnormal LPS was supported by three observations. (i) All of seven FOR mutants derived from sublines of $S$. typhimurium M747 I showed increased sensitivity to several antibiotics, comparable to the increases in sensitivity which result from $r f a$ mutations affecting biosynthesis of the deeper part of the LPS core (Roantree et al. 1969; Schlecht \& Schmidt, 1969). (ii) All of four FOR mutants tested by transduction mapped in the short $c y s E-p y r E$ segment of the linkage map, known to include nearly all the so-far identified $r f a$ genes, concerned with LPS core structure (Kuo \& Stocker, I972). (iii) One of seven FOR mutations in the $S$. typhimurium M747 I line caused complete or nearly complete loss of mouse virulence, and this loss of virulence travelled with FOR character in transduction to strain LT7 cysE.

The presence of some completed core chains in the LPS of FOR mutants is indicated by two kinds of evidence. (i) FOR mutants retain the parental $\mathrm{O}$ antigens and sensitivity to $\mathrm{O}$-specific phages. This shows the presence of $\mathrm{O}$ chains in their LPS, and it is believed that $O$ chains can be attached only to completed core chains. (ii) Many mutants isolated from FOR strains by selection for resistance to phage $\mathrm{P}_{22}$ were sensitive to FO phage, and so inferred to have some FO receptor (i.e. complete core chains) in their LPS. The phagesensitivity patterns of such FO-sensitive mutants approximated either the ' $\mathrm{R}$-sens' or the 'Zsr' (Wilkinson et al. 1972) characteristic, respectively, of mutants making complete core chains uncapped by $\mathrm{O}$ units or bearing only single $\mathrm{O}$ units. Presumably such FO-sensitive mutants of FOR strains have secondary mutations either preventing the addition of $O$ units to core chains or preventing the polymerization of $O$ units, so that complete core chains which were masked by long O chains in the LPS of the FOR parent become accessible to FO phage.

Two kinds of LPS defect might be expected to cause a reduction in number (but not total absence) of completed core chains. (i) A partial defect in some enzymic activity involved in the formation of a unit of the main chain of the core oligosaccharide. (ii) A defect, complete or incomplete, in some enzymic activity involved in a modification of a main-chain unit, if the absence of the modification impeded or prevented the addition of some distal unit(s) of the main chain. Such a modification might consist of the addition of a sugar branch or of a phosphate group to some unit of the core, as in the case of a rfaP mutant of S. minnesota, in which failure to phosphorylate a heptose unit of the core prevents addition of core units distal to glucose II (Mühlradt, I97I). These two sorts of core defect have previously been proposed to explain the properties of mutants termed part-rough class $D$, of phenotype intermediate between rough and smooth and due to mutation in or near the main $r f a$ cluster (Naide et al. 1965; Gemski \& Stocker, 1967; Wilkinson et al. 1972). Class D part-rough mutants resemble FOR mutants by their resistance to FO phage, but differ by greatly diminished sensitivity to smooth-specific phages and by sensitivity to various rough-specific phages. Members of subclass D-I, exemplified by strain SL733, are thought to be $r f a K$ mutants deficient in addition of the $N$-acetylglucosamine branch unit to the glucose II of the core chain because methylation analysis of the LPS of strain SL733 indicates the presence of many glucose II units lacking the $N$-acetylglucosamine unit at position 2 (Hellerqvist \& Lindberg, I97I). Possibly a leaky defect at $r f a K$ would produce an FOR phenotype, instead of the part-rough D-I phenotype caused by a presumably complete defect in strain SL733. 
Other FOR mutants may perhaps have leaky mutations at other $r f a$ loci previously recognized as sites of mutations causing rough or class $D$ part-rough phenotypes. The more-orless identified $r f a$ loci between $c y s E$ and $p y r E$ include $r f a G$ (close to $p y r E$ ), $r f a F$ and $r f a D$ (close to $c y e E$ ) and $r f a$ (R-res-1), $r f a$ (R-res-2) and $r f a L$ (about midway between $c y s E$ and pyrE) (Kuo \& Stocker, 1972; Lehmann et al. 1973). Four genes determining FOR phenotype were co-transduced at frequencies $>\mathrm{I} \%$, with cysE and also with pyrE (Table 2). As the frequency of co-transduction of $c y s E$ and $p y r E$ is only about $\mathrm{I} \%$ this indicates that the affected loci are between $c y s E$ and $p y r E$, but the data are insufficient to indicate their positions within this segment. As neither their phenotypic traits nor genetic analysis permit assignment of the mutant loci of FOR mutants to previously recognized $r f a$ loci, we propose $r f a(\mathrm{FOR})$ as an interim designation for all $r f a$ loci whose mutation may cause the FOR phenotype.

The increases in sensitivity to several antibiotics of all seven FOR mutants derived from S. typhimurium M747 I resembled those found in $r f a$ strains of Salmonella species with various defects deep in the LPS core (Roantree et al. 1969; Schlecht \& Schmidt, 1969). One FOR mutant, SL4505, showed greater increases in sensitivity than the rest, in particular a tenfold increase in sensitivity to erythromycin. Analogy with the similar changes observed in $r f a F$ transductants (Roantree et al. 1969; Roantree, unpublished) suggests that strain SL4505 may have a defect in the heptose region of the LPS core. Four mutants, SL4805, SL4806, SL4807 and SL4808, all showed the same, rather small, increases in sensitivities. The similar changes seen in $r f a($ R-res-I $)$ transductants suggest that these four mutants may all have lesions in the glucose II part of the core. The other two FOR mutants, SL4504 and SL4804, were intermediate in their degree of increased sensitivities, and by a similar argument may have core defects external to heptose but proximal to the glucose II unit of the core (Fig. I).

The non-virulence of FOR mutant SL4505 was also characteristic of LT7 derivatives made FOR by co-transduction of an $r f a$ gene from SL4505, and we take this as good evidence that the mutation of SL 4505 by alteration of LPS core structure causes both FOR phenotype and loss of virulence. It is noteworthy that this $S$. typhimurium strain, which would be considered to be smooth by the generally used criteria, has an LPS alteration determining complete loss of mouse virulence, as well as resistance to FO phage. Mutant SL4505 differed from the other six FOR mutants derived in strain M747I in two respects: loss of virulence, and greatly increased sensitivity to erythromycin. Both properties perhaps reflect altered outer membrane permeability resulting from an unidentified defect in the structure of the deep part of the LPS core, since LPS is believed to be a component of the outer membrane.

This investigation was supported by grant no. AIo7I68 from the National Institute of Allergy and Infectious Diseases, Department of Health, Education and Welfare. D.G.M. held a postdoctoral fellowship from the C. F. Aaron Fellowship Fund, Stanford University School of Medicine.

\section{REFERENCES}

Gemski, P. \& Stocker, B. A. D. (I967). Transduction by phage P22 in nonsmooth mutants of Salmonella typhimurium. Journal of Bacteriology 93, I 588-I 597.

Hellerqvist, C. G. \& LindberG, A. A. (1971). Structural studies of the common-core polysaccharide from Salmonella typhimurium. Carbohydrate Research 16, 39-48.

Kallings, L. O. (1967). Sensitivity of various Salmonella strains to Felix O-I phage. Acta pathologica et microbiologica scandinavica 70, 446-460.

KrishnapILlaI, V. (197I). UDPgalactose-epimerase deficiency in Salmonella typhimurium and its correction by plasmid-borne galactose genes of E. coli K I2: effects on mouse virulence. Infection and Immunity 4 , $177-188$. 
Krishnapillai, V., MacPhee, D. G. \& Stocker, B. A. D. (1971). Properties of a Salmonella typhimurium mutant with an incomplete deficiency of UDP galactose-4-epimerase. Journal of Bacteriology 107, I $55-16 \mathrm{I}$.

Kuo, T.-T. \& Stocker, B. A. D. (I972). Mapping of rfa genes in Salmonella typhimurium by ES 8 and P22 transduction and by conjugation. Journal of Bacteriology 112, 48-63.

Lehmann, V., Hämmerling, G., Nurminen, M., Minner, I., Ruschmann, E., Lüderitz, O., Kuo, T.-T. \& Stocker, B. A. D. (1973). A new class of heptose-defective mutant of Salmonella typhimurium. European Journal of Biochemistry 32, 268-275.

LindBerg, A. A. (1973). Bacteriophage receptors. Annual Review of Microbiology 27, 205-24I.

Lindberg, A. A. \& Hellerqvist, C. G. (1971). Bacteriophage attachment sites, serological specificity and chemical composition of the lipopolysaccharides of semirough and rough mutants of Salmonella typhimurium. Journal of Bacteriology 105, 57-64.

Lindberg, A. A. \& Holme, T. (I969). Influence of O side chains on the attachment of the Felix O-I bacteriophage to Salmonella bacteria. Journal of Bacteriology 99, 5I3-5I9.

Lindberg, A. A. \& Svensson, S. (i975). Salmonella typhimurium mutations conferring resistance to Felix O phage without loss of smooth character: phage attachment and immunochemical and structural analyses of lipopolysaccharides. Journal of General Microbiology 87, 1 I-I9.

MacPhee, D. G. \& Stocker, B. A. D. (1969). Suppression of amber and ochre mutants in Salmonella typhimurium by a mutant $\mathrm{F}^{\prime}$ - I-gal factor carrying an ochre suppressor gene. Journal of Bacteriology roo, $240-246$.

Morgenroth, A. \& Duguid, J. P. (I 968). Demonstration of different mutational sites controlling rhamnose fermentation in FIRN and non-FIRN rha ${ }^{-}$strains of Salmonella typhimurium. Genetical Research $\mathbf{r r}$, I 5 I-169.

MühlRadt, P. F. (197I). Biosynthesis of Salmonella lipopolysaccharide. Studies on the transfer of glucose, galactose and phosphate to the core in a cell-free system. European Journal of Biochemistry 18, $20-27$.

Naide, Y., Nikaido, H., Mäkelä, P. H., Wilkinson, R. G. \& Stocker, B. A. D. (I965). Semirough strains of Salmonella. Proceedings of the National Academy of Sciences of the United States of America 53, 147-I53.

Roantree, R. J., Kuo, T.-T., MacPhee, D. G. \& Stocker, B. A. D. (1969). Effect of various rough lesions in Salmonella typhimurium upon sensitivity to antibiotics. Bacteriological Proceedings, p. 79.

Sanderson, K. E., Ross, H., Ziegler, L. \& Mäkelä, P. H. (I972). F+ , Hfr and F' strains of Salmonella typhimurium and Salmonella abony. Bacteriological Reviews 38, 608-637.

SANDERSON, K. E. \& SAEED, H. (1972). Insertion of the F factor into the cluster of $r f a$ (Rough A) genes of Salmonella typhimurium. Journal of Bacteriology 112, 64-73.

SCHLecht, S. \& SCHмIDT, G. (1969). Möglichkeiten zur Differenzierung von Salmonella-R-Formen mittels Antibiotica und antibakterieller Farbstoffe. Zentralblatt für Bakteriologie, Parasitenkunde, Infektionskrankheiten und Hygiene (Abteilung I) 212, 505-5I I.

Steward, J. P., Collis, L. R. \& Roantree, R. J. (1966). Effects of immunization of guinea pigs lacking bactericidal antibody against Salmonella enteritidis. Journal of Immunology 97, 224-230.

SubbiaH, T. V. (1963). Genetics of virulence in Salmonella typhimurium; mapping of rough and other loci concerned. Ph.D. Thesis, University of London.

Wilkinson, R. G., Gemski, P. \& STOCKer, B. A. D. (1972). Non-smooth mutants of Salmonella typhimurium: differentiation by phage sensitivity and genetic mapping. Journal of General Microbiology 70, 527-554. 